Article

\title{
(Perylene) $)_{3}-\left(\mathrm{TCNQF}_{1}\right)_{2}$ : Yet Another Member in the Series of Perylene-TCNQF $F_{x}$ Polymorphic Charge Transfer Crystals
}

\author{
Tommaso Salzillo ${ }^{1,2, *} \mathbb{0}$, Raffaele G. Della Valle ${ }^{1}\left(\mathbb{D}\right.$, Elisabetta Venuti $^{1}$, Aldo Brillante ${ }^{1}$, \\ Gabriele Kociok-Köhn ${ }^{3}$, Daniele Di Nuzzo ${ }^{4}$, Matteo Masino ${ }^{5, *(D)}$ and Alberto Girlando ${ }^{5, *}$ \\ 1 Dipartimento di Chimica Industriale “Toso Montanari”, Università di Bologna and INSTM UdR Bologna, \\ Viale Risorgimento, 40136 Bologna, Italy; raffaele.dellavalle@unibo.it (R.G.D.V.); \\ elisabetta.venuti@unibo.it (E.V.); aldo.brillante@unibo.it (A.B.) \\ 2 Department of Molecular Nanoscience and Organic Materials, Institut de Ciència de Materials de \\ Barcelona (ICMAB-CSIC) and (CIBER-BBN), Campus de la UAB, 09193 Bellaterra, Spain \\ 3 Material and Chemical Characterization facility (MC2), University of Bath, Claverton Down, Bath BA2 7AY, \\ UK; chsgk@bath.ac.uk \\ 4 Cavendish Laboratory, University of Cambridge, JJ Thomson Avenue, Cambridge CB3 OHE, UK; \\ dd467@cam.ac.uk \\ 5 Dipartimento di Scienze Chimiche, della Vita e della Sostenibilità Ambientale (SCVSA), Università di Parma \\ and INSTM-UdR Parma, Parco Area delle Scienze 17/a, 43124 Parma, Italy \\ * Correspondence: tommaso.salzillo@unibo.it (T.S.); matteo.masino@unipr.it (M.M.); \\ alberto.girlando@unipr.it (A.G.)
}

Received: 18 February 2020; Accepted: 4 March 2020; Published: 6 March 2020

\begin{abstract}
The 3:2 Charge Transfer (CT) co-crystal (Perylene) $)_{3}\left(\mathrm{TCNQF}_{1}\right)_{2}$ is grown by the Physical Vapor Transport (PVT) method, and characterized structurally and spectroscopically. Infrared analysis of the charge sensitive modes reveals a low degree of charge transfer (less than 0.1 ) between donor and acceptor molecules. The crystal is isostructural to the other 3:2 CT crystals formed by Perylene with $\mathrm{TCNQF}_{2}$ and $\mathrm{TCNQF}_{4}$, whereas such stoichiometry and packing is not known for the CT crystals with non-fluorinated TCNQ. The analysis of the isostructural family of 3:2 Perylene-TCNQF ${ }_{x}(x=1,2,4)$ co-crystal put in evidence the role of weak $\mathrm{F} \cdots \mathrm{HC}$ bonding in stabilizing this type of structure
\end{abstract}

Keywords: charge transfer crystals; X-ray diffraction; vibrational spectroscopy

\section{Introduction}

Recently, the study of charge transfer (CT) crystals has been reinvigorated by its potential applications in organic electronics [1,2], due to the possibility of having both $n$ - and $p$-type behaviors in organic field effect transistors (OFETs) [3-5]. The CT interaction between the frontier $\pi$-orbitals of the electron Donor (D) and Acceptor (A) molecules is generally the predominant intermolecular interaction in a solid state, so that many properties, including mobility, have strong directional characters. The packing is indeed characterized by the presence of stacking of the involved $\pi$-molecules, hence, the name of "quasi 1-dimensional" crystals for these systems.

Because of the focus on the role of the CT interaction, the occurrence of polymorphism in CT crystals has been sort of neglected, at least in comparison with one-component $\pi-$ molecular semiconductors [6]. However, the above mentioned increased research activity in the field of CT crystal, polymorphism and its effect on the physical properties have started to emerge, even for the prototypical ambipolar system DBTTF-TCNQ [7,8]. As a matter of fact, the family of co-crystals made up of Perylene and F-substituted Tetracyanoquinodimethane (TCNQF ${ }_{x}$ ) exhibits such a large variety 
of different packings and stoichiometry as to be considered as a paradigmatic example of the old sentence: "the number of forms known for a given compound is proportional to the time and energy spent in research on that compound" [9]. Three different polymorphs with 1:1 ratio of D to A $(\alpha, \beta$ and $\gamma$ ) are known for the crystals formed by Perylene with unsubstituted TCNQ [10], accompanied by two "stoichiomorphs" with 2:1 and 3:1 composition [11,12]. Perylene and TCNQF 2 form two types of crystals, with 1:1 and 3:2 stoichiometry. In addition to these two stoichiometries, $\mathrm{TCNQ}_{4}$ also forms a solvated CT crystal with the inclusion of toluene [13]. This wide variety of packings allows one to try to correlate physical properties with the packing motif $[11,14]$, and suggests that the role of crystal engineering in building up the CT co-crystals with the desired properties is not limited to the choice of the D/A pair with the proper ionization potential and electron affinity, but that other types of intermolecular interactions have to be taken into account.

In this paper we present the structural and spectroscopic characterization of a 3:2 CT crystal of Perylene-TCNQF 1 , which adds to the already known 1:1 system [15]. This new system is isostructural to (Perylene $)_{3}-\left(\mathrm{TCNQF}_{2}\right)_{2}$ and to (Perylene $)_{3}-\left(\mathrm{TCNQF}_{4}\right)_{2}[13]$, so that all the three F-substituted TCNQs give rise to two different co-crystals with 1:1 and 3:2 D/A ratio, while no 3:2 co-crystals of Perylene with unsubstituted TCNQ are known. We also report on the attempt to include TCNQ in a 3:2 system together with $\mathrm{TCNQF}_{2}$. Analysis of the structures of the three 3:2 Perylene- $\mathrm{TCNQF}_{\mathrm{x}} \mathrm{CT}$ crystals allowed us to underline the role of the weak $\mathrm{F} \cdots \mathrm{HC}$ bonding in stabilizing this type of packing.

\section{Materials and Methods}

\subsection{Sample Preparation and Crystal Growth}

Perylene (Sigma Aldrich (St. Louis, MI, USA), purity 99\%), TCNQ (Sigma Aldrich, purity 98\%), TCNQF 1 (Tokyo Chemical Industry (Tokyo, Japan), purity 98\%) and TCNQF 2 (Tokyo Chemical Industry, purity $98 \%$ ) were used as purchased. All solvents (from Aldrich (St. Louis, Missouri, USA)) were of spectroscopic grade. The single crystals of the binary system Perylene- $\mathrm{TCNQF}_{1}$ and of the supposedly ternary system Perylene- $\mathrm{TCNQF}_{2}[\mathrm{TCNQ}]$ were obtained by the Physical Vapor Transport (PVT) method. A quartz ampoule of $100 \mathrm{~mm}$ length and $10 \mathrm{~mm}$ diameter was cleansed with soap, distilled water, isopropanol and acetone, in this order, and then left to dry overnight at $110^{\circ} \mathrm{C}$. For the crystal growths, the ampoule was loaded with equimolar amounts of the parent compounds, namely, Perylene and $\mathrm{TCNQF}_{1}$ in the first case, and Perylene, TCNQ and $\mathrm{TCNQF}_{2}$ in the second case. The ampoule was then evacuated in $\mathrm{N}_{2}$ to a final pressure of $10^{-4}$ mbar, sealed with an oxygen-natural gas burner, and placed in a two-zone furnace. For Perylene-TCNQF $170^{\circ} \mathrm{C}$ and $110^{\circ} \mathrm{C}$ at the hot and cold end of the apparatus, respectively; for Perylene-TCNQF 2 TCNQ], temperatures of $178{ }^{\circ} \mathrm{C}$ and $128^{\circ} \mathrm{C}$ were selected for the same setup (see Figure 1).

The potassium salt of $\mathrm{TCNQF}_{1}\left(\mathrm{~K}^{+} \mathrm{TCNQF}_{1}^{-}\right)$was prepared following the experimental procedure described by Melby et al. [16], using the reaction of the iodide with $\mathrm{TCNQF}_{1}$. When the hot solutions of potassium iodide and $\mathrm{TCNQF}_{1}$ in acetonitrile were mixed, the potassium salt precipitated as a dark purple crystalline solid with a needle-like morphology. $\mathrm{TCNQF}_{1}$ and potassium iodide were mixed in ratio 2:3 using the excess of metal iodide to scavenge the iodine by-product as $\mathrm{I}^{3-}$.

\subsection{X-ray Diffraction Measurements}

Single crystal diffraction data for the Perylene-TCNQF ${ }_{1}$ and Perylene-TCNQF 2 [TCNQ] 3:2 structures were collected at $150 \mathrm{~K}$ on a Nonius Kappa CCD diffractometer (Netherlands)with graphite mono-chromated Mo-K $\alpha$ radiation, $\lambda=0.71073 \AA$. Unit cell determination, data scaling, and corrections for Lorentz and polarization effects were performed with Denzo-SMN [17]. A symmetry-related (multi-scan) absorption correction was applied. Structure solution, followed by full-matrix least-squares refinement, was performed using the WINGX-v2014.1 [18] suite of programs throughout. Hydrogen atoms were placed onto calculated positions and refined using a riding model. Crystallographic data 
for the reported structures have been deposited with the Cambridge Crystallographic Data Centre (CCDC), reference numbers CCDC 1963867 and 1963868.

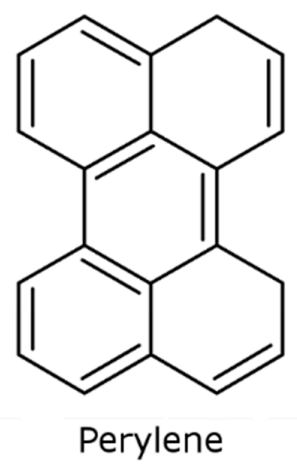

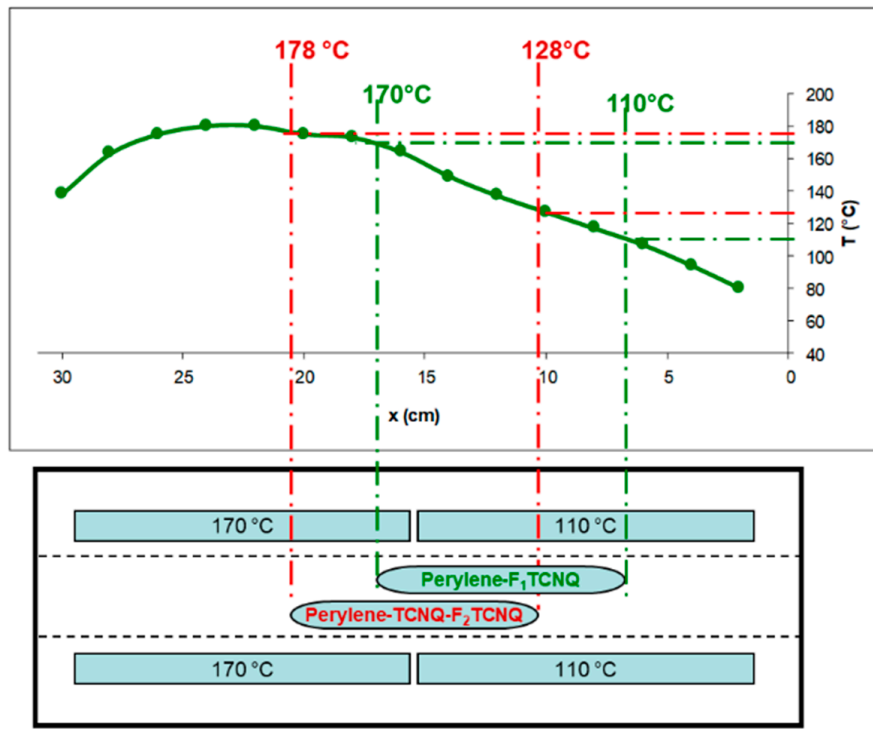<smiles>N#CC(C#N)=c1ccc(=C(C#N)C#N)cc1</smiles>
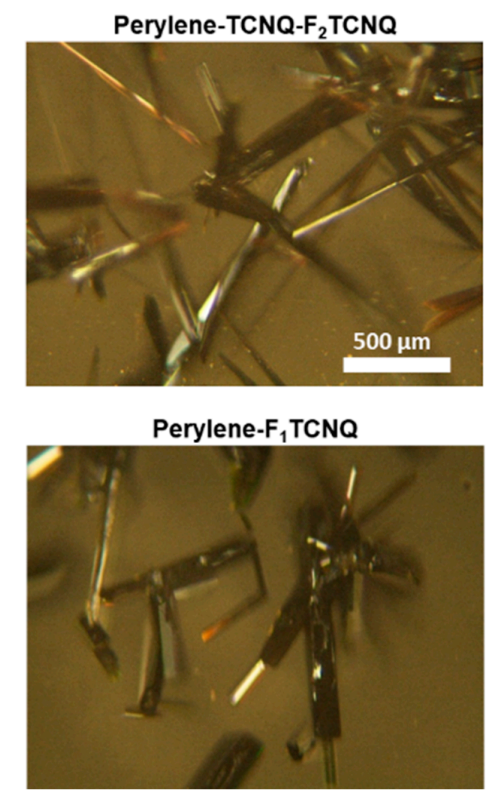

Figure 1. Chemical formulas (top), scheme of the PVT crystal growth technique (bottom left), and photographs of the grown crystals (bottom right).

\subsection{Spectroscopic Measurements}

Infrared (IR) spectra of the CT crystals were recorded with a Bruker IFS66 (Ettlingen, Germany). Fourier transform IR (FT-IR) spectrometer coupled to an IR microscope Hyperion 1000. The spectrometer is equipped with a liquid nitrogen cooled mercury cadmium telluride (MCT) detector. The instrument setup allows for reflection and transmission measurements with polarized light.

The Raman spectra were recorded with a Horiba Jobin Yvon T64000 triple gratings Raman spectrometer equipped with the appropriate interferential filter and coupled to an Olympus BX40 microscope (Shinjuku, Tokyo, Japan) with $50 \times$ or $100 \times$ objectives. The $647.1 \mathrm{~nm}$ excitation line from a Kripton ion $(\mathrm{Kr}+)$ laser was used as light source, wavelength long enough to prevent fluorescence emission of the studied systems.

\section{Results}

\subsection{Crystal Structure}

The binary system Perylene-TCNQF 1 obtained by the PVT method in an open tube using inert gas as carrier transport is known to crystallize in a monoclinic structure with mixed stack DA arrangement in a 1:1 ratio [15]. By growing the crystals by PVT in a closed ampoule (see Section 2.1) we obtain 
the Perylene-TCNQF 1 stochiomorph in a 3:2 D/A ratio. This CT crystal crystallizes in the triclinic space group P-1 and is characterized by a slightly dimerized ... DADAD ... . stack along the a-axis and trimeric (DAD) units arranged in a zig-zag pattern along the c-axis (Figure 2). The crystal is isostructural to the other two 3:2 CT crystals formed by Perylene with $\mathrm{TCNQF}_{2}$ and $\mathrm{TCNQF}_{4}$, also grown by PVT in closed ampoule [13]. A comparison of the basic structural parameters of the three CT crystals is given in Table 1.
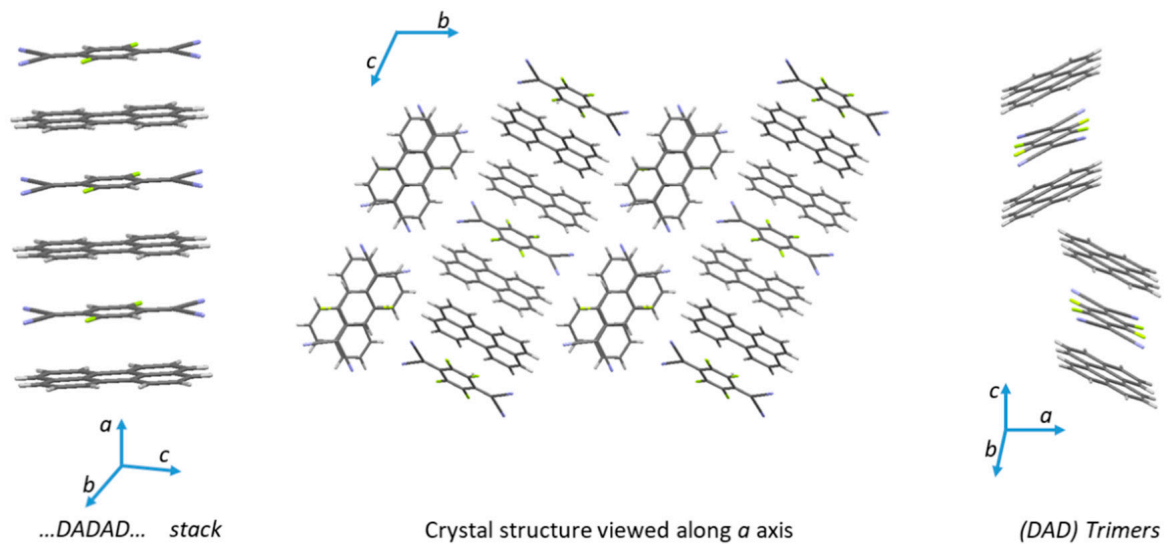

(DAD) Trimers

Figure 2. Crystal structure of (Perylene $)_{3}-\left(\mathrm{TCNQF}_{1}\right)_{2}$.

Table 1. Basic structural parameters of the three isostructural CT crystals Perylene-TCNQF $3: 2$.

\begin{tabular}{cccc}
\hline & TCNQF $_{\mathbf{1}} \mathbf{1}^{-}$ & TCNQF $_{\mathbf{2}}{ }^{2}$ & TCNQF $_{\mathbf{4}}{ }^{\mathbf{2}}$ \\
\hline Stoichiometry & $3: 2$ & $3: 2$ & $3: 2$ \\
Space group & $\mathrm{P}-1$ & $\mathrm{P}-1$ & $\mathrm{P}-1$ \\
Temperature $(\mathrm{K})$ & $150(2)$ & $150(2)$ & $150(2)$ \\
$a(\AA)$ & $7.2254(2)$ & $7.2307(1)$ & $7.2066(2)$ \\
$b(\AA)$ & $19.1275(4)$ & $19.1407(3)$ & $19.0974(6)$ \\
$c(\AA)$ & $22.3203(6)$ & $22.2723(5)$ & $22.4050(7)$ \\
$\alpha\left(^{\circ}\right)$ & $112.2658(14)$ & $112.2834(8)$ & $111.8014(14)$ \\
$\beta\left(^{\circ}\right)$ & $90.2346(10)$ & $90.1900(8)$ & $90.0472(17)$ \\
$\gamma\left({ }^{\circ}\right)$ & $94.0322(15)$ & $93.7599(12)$ & $94.1283(17)$ \\
$V\left(\AA^{3}\right)$ & $2846.09(13)$ & $2844.85(9)$ & $2854.3(15)$ \\
$Z$ & 2 & 2 & 2
\end{tabular}

[a] R1 $=\Sigma\|\mathrm{Fo}|-| \mathrm{Fc}\| / \Sigma|\mathrm{Fo}|,[\mathrm{b}] \mathrm{wR} 2=\{\Sigma[\mathrm{w}(\mathrm{Fo} 2-\mathrm{Fc} 2) 2] / \Sigma[\mathrm{w}(\mathrm{Fo} 2) 2]\} 1 / 2^{1}$ This work. ${ }^{2}$ From Ref. [13].

The slightly dimerized stack displays an average D-A distance of $3.32 \AA$ and an intrinsic disorder of the $\mathrm{F}$ atoms. The F occupancy was refined up to the best $\mathrm{R}$ value, then it was kept fixed in the final stage to avoid non-integer numbers, with practically no change in the $\mathrm{R}$ value. We get $80 \%$ overall occupancy factor in positions 2 and 5 of the TCNQ ring. The DAD trimers are arranged quasi perpendicularly to the ... DADAD ... . stack, with the $\mathrm{TCNQF}_{1}$ molecules residing on inversion center, with a random disorder of the $\mathrm{F}$ atoms, approx. $25 \%$ of occupancy on each position. The average D-A distance within the trimers $(3.26 \AA)$ is considerably shorter than that in the stacks. Furthermore, the trimeric units display a tilted angle between the Perylene units of $48.33^{\circ}$.

We have also attempted to obtain a ternary crystal made by the combination of Perylene with a 1:1 mixture of TCNQ and TCNQF 2 . The crystal is isostructural to the just examined 3:2 Perylene-TCNQF but the X-ray analysis did not show clear evidence of the presence of the non-fluorinated TCNQ. The refinement gave an acceptable R-factor by assuming that the crystal was actually twinned, and we obtained only a slight improvement to the final $\mathrm{R}$ of $10.4 \%$ by also assuming that some TCNQ goes into the DADAD stack, whereas its presence in the trimers is very dubious. The final structural parameters (CCDC 1963868) are the same, within the experimental error, as those of pure (Perylene $)_{3}\left(\mathrm{TCNQF}_{2}\right)_{2}$ (Table 1), and also the spectroscopic measurements did not show evidences of TCNQ. We conclude that non-fluorinated TCNQ, at most, enters the crystal as an impurity, so that we label the system as 3:2 Perylene- $\mathrm{TCNQF}_{2}[\mathrm{TCNQ}]$, and shall not discuss it anymore here. 


\subsection{Determination of the Degree of CT}

One of the key parameters characterizing the ground state properties of CT crystals is the ionicity, i.e., the degree of charge transfer from D to A. The degree of CT essentially depends on the donor/acceptor characteristics of the molecular components, i.e., the ionization potential and the electron affinity, and on the packing arrangement inside the crystal, which affects the Madelung energy. Therefore, if like in the present case, we have a series of isostructural crystals with differently fluorinated $\mathrm{TCNQF}_{\mathrm{x}}$ having increasing electron affinities, we expect to find increasing $\rho$ values. Vibrational spectroscopy is a well-established method to estimate the degree of charge transfer in CT crystals by looking at the frequencies of the so-called "charge sensitive" vibrational modes, i.e., the modes with frequencies strongly affected by the charge residing on the molecular site $[19,20]$. Such modes must be singled out by studying the vibrational spectra of the neutral and fully ionic molecule. Raman is not reliable for mixed stack CT crystals like the present ones, as the frequencies of totally symmetric modes may be affected by the coupling with the CT system [19,20]; thus, we shall limit ourselves to examining the only IR active modes in detail.

Since the strong acceptor TCNQ skeleton is easily ionized, the determination of $\rho$ in binary systems is generally based on its charge sensitive modes. These have been already identified for $\mathrm{TCNQ}, \mathrm{TCNQF}_{2}$ and $\mathrm{TCNQF}_{4}$ [[13] and references therein]. Instead, to the best of our knowledge, a detailed study of $\mathrm{TCNQF}_{1}$ vibrations is still missing, even though this molecule is increasingly used in the formation of CT crystals [21]. For this reason, the details of the analysis are given here. To identify the charge sensitive modes of $\mathrm{TCNQF}_{1}$ we have measured the IR absorption spectra of the neutral molecule and its anion $\mathrm{TCNQF}_{1}{ }^{-}$in the $\mathrm{K}^{+} \mathrm{TCNQF}_{1}{ }^{-}$salt. The spectra are reported in Figure 3. The full analysis and the assignment of the IR spectra have been carried out with the help of quantum chemical calculations, the details of which are reported in the Supplementary Materials. In Table S1, we report the harmonic frequencies, infrared intensities and normal mode eigenvectors of the most important vibrational modes calculated by the DFT method using the B3LYP hybrid functional and 6.31G $(\mathrm{d}, \mathrm{p})$ basis set for both neutral and ionic $\mathrm{TCNQF}_{1}$.

The three vibrational bands marked in Figure 3 with full vertical lines have been found to show the largest ionicity frequency shifts $(\Delta)$ for the $\mathrm{TCNQF}_{1}$ molecule. In terms of their harmonic eigenvectors, modes $v_{1}$ and $v_{2}$ correspond to the in-phase and the out-of-phase stretching of the two $C=C$ bonds of the TCNQ ring, while mode $v_{3}$ can be described as the anti-symmetric stretching of the two apical $C=C$ bonds (see Figure 3). However, while the $v_{1}$ and $v_{2}$ bands can be safely assigned in the spectra of neutral $\left(1618 \mathrm{~cm}^{-1}\right.$ and $\left.1557 \mathrm{~cm}^{-1}\right)$ and ionic TCNQF $1\left(1591 \mathrm{~cm}^{-1}\right.$ and $\left.1515 \mathrm{~cm}^{-1}\right)$, the $v_{3}$ band, occurring at $1549 \mathrm{~cm}^{-1}$ in the spectrum of the neutral molecule, shift down to $1361 \mathrm{~cm}^{-1}$ upon ionization, crossing several other vibrational modes and slightly altering its eigenvector description. Therefore we do not expect [22] that it will exhibit the linear dependence on $\rho$ necessary to estimate the degree of CT in case of intermediate ionicity. For analogous reasons, we exclude the $\mathrm{C} \equiv \mathrm{N}$ antisymmetric stretching modes (inset of Figure 3), which also show appreciable ionicity frequency shift, but are potentially affected by the molecular environment like electrostatic potential, hydrogen and halogen bonding [23,24]. In the estimate of $\rho$ for CT crystals involving TCNQF 1 we mainly rely on the $v_{1}$ and $v_{2}$ modes, exhibiting an ionicity red-shift of $27 \mathrm{~cm}^{-1}$ and $42 \mathrm{~cm}^{-1}$, respectively, and use the $v_{3}$ as a consistency check.

In Figure 4 we report the IR absorption spectra of the (Perylene $)_{3}-\left(\mathrm{TCNQF}_{1}\right)_{2} \mathrm{CT}$ crystal polarized along two mutually perpendicular directions, one with the electric vector along the a-axis, namely, along the ... DADAD ... stack (red curve), and the other perpendicular to it (blue curve), namely, along the alignment direction of the (DAD) trimers. In the latter spectrum, we then observe the in-plane modes of the molecules within the stack, and we single out the three above-mentioned charge sensitive bands occurring at 1617,1554 and $1543 \mathrm{~cm}^{-1}$, which are only slightly shifted with respect to the bands of the neutral TCNQF 1 (Figure 3 and dashed black line in Figure 4). The position of these bands yields an estimate of $\rho$ of 0.04 and 0.09 for the $v_{1}$ and $v_{2}$ modes, respectively, and 0.03 for the less reliable $v_{3}$ mode. We conclude that the degree of $\mathrm{CT}$ is very small in the 3:2 Perylene-TCNQF $F_{1}$ crystal, with an estimate of $\rho$ below 0.1 , very similar to the 1:1 Perylene-TCNQ stoichiomorph [13]. Raman spectra 
also confirm the slight ionicity of the system as for the small shift of the $v_{R}(C=C)$ vibration at about $1459 \mathrm{~cm}^{-1}$ of the neutral TCNQF 1 (see Supplementary Materials, Figure S1). We conclude that the addition of just one F atom to the TCNQ skeleton does not increase the degree of CT appreciably with respect to the non-fluorinated TCNQ.
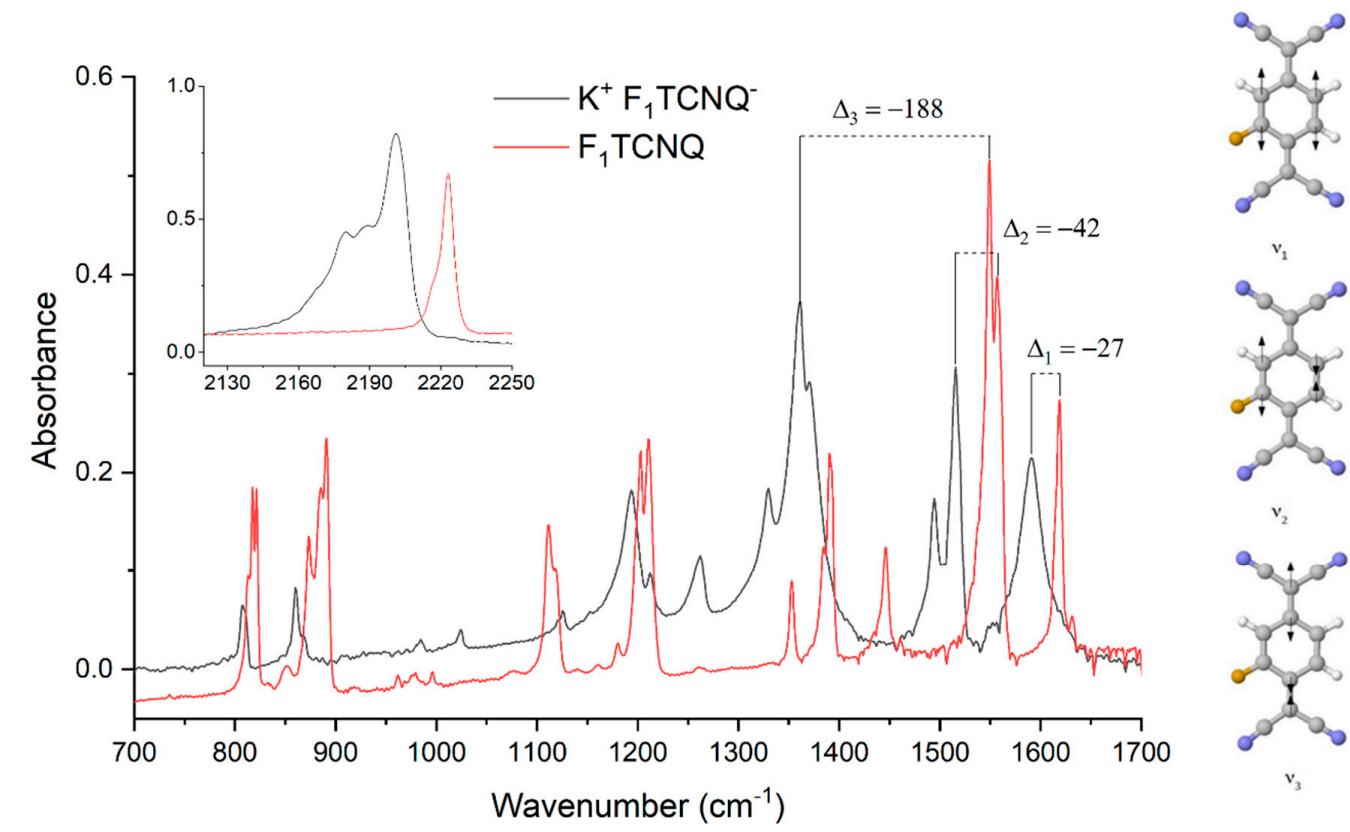

Figure 3. IR absorption spectra of $\mathrm{TCNQF}_{1}$ and $\mathrm{K}^{+} \mathrm{TCNQF}_{1}{ }^{-}$powders (left) and schematics of the charge sensitive vibrational modes (right).

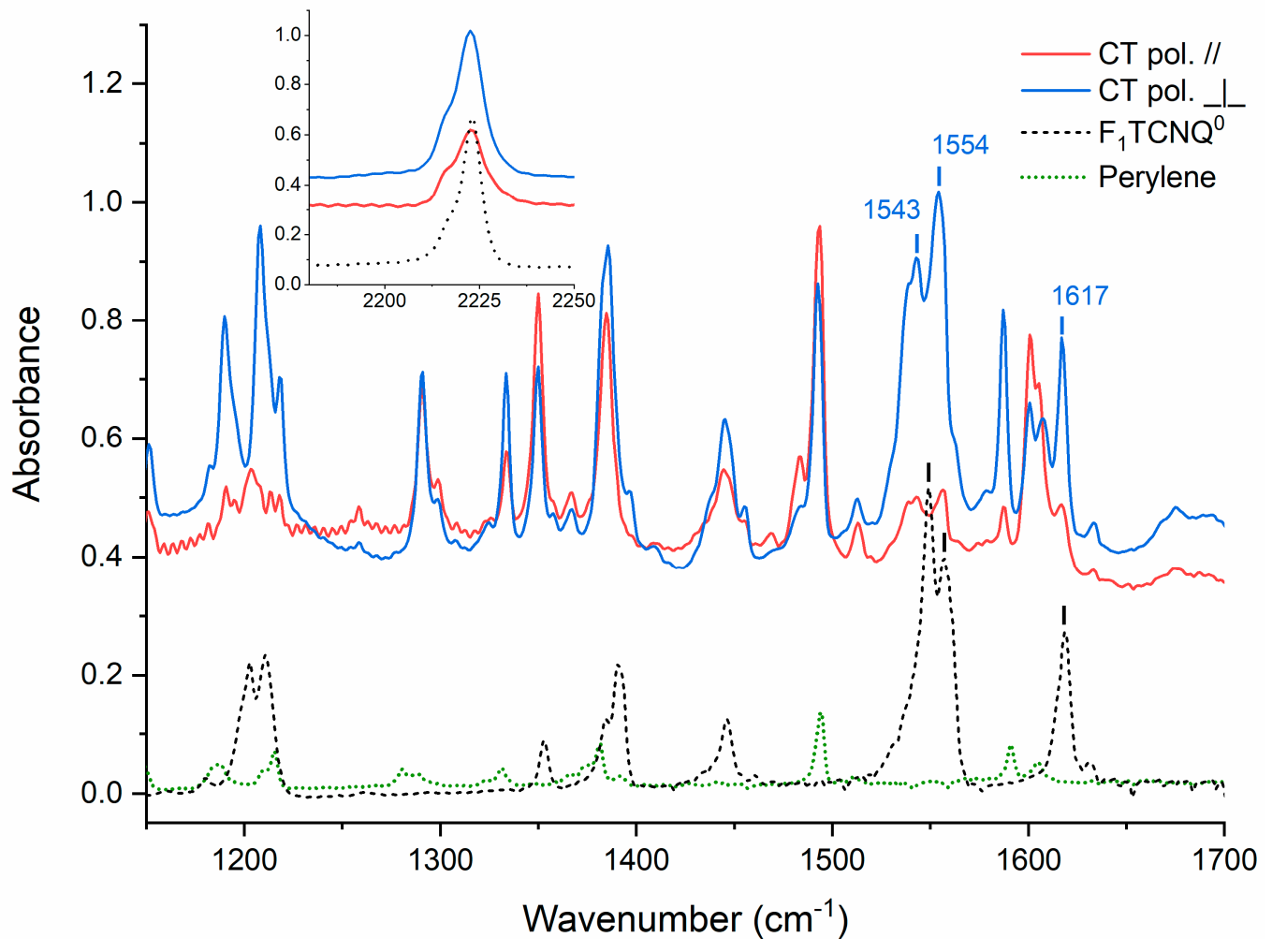

Figure 4. Polarized IR absorption spectra of Perylene-TCNQF 1 3:2 CT crystal. 


\section{Discussion and Conclusions}

The Perylene-TCNQF 1 3:2 structure presented here completes the series of CT crystals formed by Perylene with $\mathrm{TCNQF}_{\mathrm{x}}$, in the sense that all the co-crystals containing fluorinated TCNQ crystallize either in a stacked structure ... DADA ... with D/A 1:1 ratio, or in isostructural 3:2 co-crystals where the stack is accompanied by (DAD) lateral trimers. As we have already noted in the Introduction, Perylene forms with TCNQ several CT co-crystals, with 1:1, 2:1 and 3:1 DA ratios [10,11,13,15,25,26], but none with the $3: 2$ ratio and the associated triclinic packing. Furthermore, the attempt to insert non-fluorinated TCNQ into the 3:2 structure of $\mathrm{TCNQF}_{2}$ essentially failed; although it may be related to growth conditions, different sublimation temperatures etc. Finally, in our experience, the 1:1 co-crystal Perylene-TCNQF 1 is obtained more easily than the 3:2 reported here for the first time, but the opposite is true for Perylene-TCNQF 4 [13], whereas in the case of Perylene-TCNQF 2 in solution, an equilibrium is established between the two stoichiomorphs [27].

The above empirical observations suggest that the 3:2 packing is favored by the presence of $\mathrm{F}$ atoms. A hint of the reason is given by the fact that in the 3:2 structure of both $\mathrm{TCNQF}_{1}$ and $\mathrm{TCNQF}_{2}$, the $\mathrm{F}$ atoms of the stacked TCNQFx preferentially occupy the 2,5 position. By looking at the structure (Perylene $)_{3}(\text { TCNQF4) })_{2}$ in Figure 5 we indeed notice that within the ... DADAD ... stack, only the $\mathrm{F}$ atoms in 2.5 positions have short contact with the Perylene trimers. There are also short F $\cdots \mathrm{HC}$ inter-trimer contacts, so as to stabilize 2-dimensional lateral trimer networks, but in this case, all the four $\mathrm{F}$ positions are equally involved.

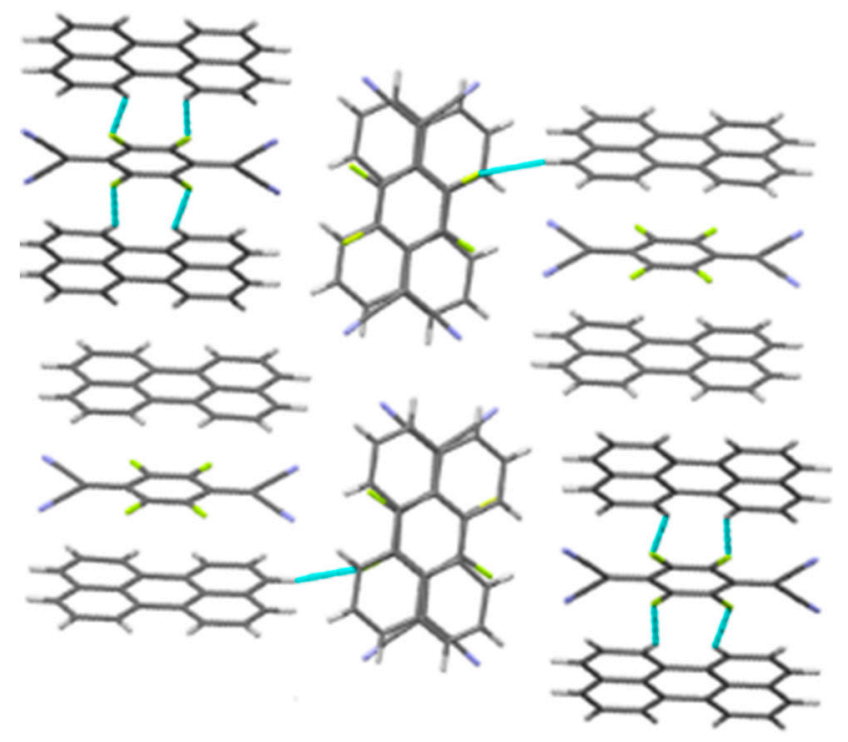

Figure 5. Structure of (Perylene $)_{3}\left(\mathrm{TCNQF}_{4}\right)_{2}$ viewed along the a-axis. F ${ }^{\cdots}$ H contacts are evidenced in turquoise.

The present study demonstrates that the F atoms not only increase the TCNQ electron affinity, but also have an active role in the crystal packing through $\mathrm{F} \cdots \mathrm{H}$ bonds. The importance of halogen bonding is of course well known in crystal engineering, although the role of weak intermolecular bonding involving $\mathrm{F}$ and $\mathrm{HC}$ is still an active matter of study [28]. Here, we have an example of the interplay of $\mathrm{F} \cdots \mathrm{HC}$ intermolecular bonding with 1-dimensional CT interaction that favors the face-to-face molecular arrangement of the $\pi$-molecules. We believe that such $\mathrm{CT} / \mathrm{F} \cdots \mathrm{HC}$ interplay is worth further investigation, as fluorinated molecules are making their way in the field of organic materials, where proper crystal engineering is of vital importance [29].

Supplementary Materials: The following are available online at http://www.mdpi.com/2073-4352/10/3/177/s1, Table S1. Frequencies, eigenvectors and infrared intensities of the most important vibrational modes; Figure S1. Raman spectra of the neutral TCNQF1(black), Perylene (green), potassium TCNQF1 salt (red) and of the charge transfer complex TCNQF1-Perylene (blue). 
Author Contributions: Conceptualization, T.S.; Data curation, M.M. and G.K.-K.; Methodology, A.B.; Writing-original draft, A.G.; Writing—review \& editing, R.G.D.V., E.V. and D.D.N. All authors have read and agreed to the published version of the manuscript.

Funding: The work has been supported by the local research funds of Bologna and Parma Universities.

Acknowledgments: The authors thank Enrico Da Como (Bath University) for helpful discussions in the early stages of this work, and Chiara Massera (Parma University) for discussion and assistance in the interpretation of the structural data.

Conflicts of Interest: The authors declare no conflict of interest

\section{References}

1. Jiang, H.; Hu, P.; Ye, J.; Zhang, K.K.; Long, Y.; Hu, W.; Kloc, C. Tuning of the degree of charge transfer and the electronic properties in organic binary compounds by crystal engineering: A perspective. J. Mater. Chem. C 2018, 6, 1884-1902. [CrossRef]

2. Sun, L.; Zhu, W.; Yang, F.; Li, B.; Ren, X.; Zhang, X.; Hu, W. Molecular cocrystals: Design, charge-Transfer and optoelectronic functionality. Phys. Chem. Chem. Phys. 2018, 20, 6009-6023. [CrossRef] [PubMed]

3. Zhu, L.; Yi, Y.; Li, Y.; Kim, E.G.; Coropceanu, V.; Brédas, J.L. Prediction of remarkable ambipolar charge-Transport characteristics in organic mixed-stack charge-Transfer crystals. J. Am. Chem. Soc. 2012, 134, 2340-2347. [CrossRef] [PubMed]

4. Goetz, K.P.; Vermeulen, D.; Payne, M.E.; Kloc, C.; McNeil, L.E.; Jurchescu, O.D. Charge-Transfer complexes: New perspectives on an old class of compounds. J. Mater. Chem. C 2014, 2, 3065-3076. [CrossRef]

5. Geng, H.; Zheng, X.; Shuai, Z.; Zhu, L.; Yi, Y. Understanding the charge transport and polarities in organic donor-acceptor mixed-stack crystals: Molecular insights from the super-exchange couplings. Adv. Mater. 2015, 27, 1443-1449. [CrossRef]

6. Bernstein, J. Polymorphism in Molecular Crystals; Oxford University Press: New York, NY, USA, 2002.

7. Goetz, K.P.; Tsutsumi, J.; Pookpanratana, S.; Chen, J.; Corbin, N.S.; Behera, R.K.; Coropceanu, V.; Richter, C.A.; Hacker, C.A.; Hasegawa, T.; et al. Polymorphism in the 1:1 Charge-Transfer Complex DBTTF-TCNQ and Its Effects on Optical and Electronic Properties. Adv. Electron. Mater. 2016, 2, 1-10. [CrossRef]

8. Salzillo, T.; Campos, A.; Mas-Torrent, M. Solution-processed thin films of a charge transfer complex for ambipolar field-effect transistors. J. Mater. Chem. C 2019, 7, 10257-10263. [CrossRef]

9. McCrone, W.C. Polymorphism in Physics and Chemistry of the Organic Solid State, Vol. II.; Fox, D., Labes, M.M., Weissenberg, A., Eds.; Interscience: New York, NY, USA, 1965; Volume II.

10. Henderson, J.; Masino, M.; Hatcher, L.E.; Kociok-Köhn, G.; Salzillo, T.; Brillante, A.; Raithby, P.R.; Girlando, A.; Da Como, E. New Polymorphs of Perylene: Tetracyanoquinodimethane Charge Transfer Cocrystals. Cryst. Growth Des. 2018, 18, 2003-2009. [CrossRef]

11. Vermeulen, D.; Zhu, L.Y.; Goetz, K.P.; Hu, P.; Jiang, H.; Day, C.S.; Jurchescu, O.D.; Coropceanu, V.; Kloc, C.; McNeil, L.E. Charge Transport Properties of Perylene - TCNQ Crystals: The Effect of Stoichiometry. J. Phys. Chem. C 2014, 118, 24688-24696. [CrossRef]

12. Ishii, K.; Yakushi, K.; Kuroda, H.; Inokuchi, H. Reflection and Photoconduction Spectra of the Single Crystals of Perylene-TCNQ 1:1 and 3:1 Molecular Complexes. Bull. Chem. Soc. Jpn. 1984, 57, 3043-3047. [CrossRef]

13. Salzillo, T.; Masino, M.; Kociok-Köhn, G.; Di Nuzzo, D.; Venuti, E.; Della Valle, R.G.; Vanossi, D.; Fontanesi, C.; Girlando, A.; Brillante, A.; et al. Structure, Stoichiometry, and Charge Transfer in Cocrystals of Perylene with TCNQ-Fx. Cryst. Growth Des. 2016, 16, 3028-3036. [CrossRef]

14. Wang, Y.; Zheng, C.; Hao, W.; Zhao, H.; Li, S.; Shen, L.; Zhu, J.; Di, C.A. Impact of Stoichiometry and Fluorine Atoms on the Charge Transport of Perylene-F4TCNQ. J. Phys. Chem. Lett. 2019, 10, 3376-3380. [CrossRef] [PubMed]

15. Hu, P.; Du, K.; Wei, F.; Jiang, H.; Kloc, C. Crystal growth, HOMO-LUMO engineering, and charge transfer degree in perylene-FxTCNQ $(x=1,2,4)$ organic charge transfer binary compounds. Cryst. Growth Des. 2016, 16, 3019-3027. [CrossRef]

16. Melby, L.R.; Harder, R.J.; Hertler, W.R.; Mahler, W.; Benson, R.E.; Mochel, W.E. Substituted Quinodimethans. II. Anion-Radical Derivatives and Complexes of 7, 7, 8, 8-Tetracyanoquinodimethan. J. Am. Chem. Soc. 1962, 84, 3374-3387. [CrossRef] 
17. Otwinowski, Z.; Minor, W. Processing of X-Ray Diffraction Data Collected in Oscillation Mode. Macromol. Crystallogr. 1997, 276, 307-326.

18. Farrugia, L.J. WinGX suite for small-molecule single-crystal crystallography. J. Appl. Crystallogr. 1999, 32, 837-838. [CrossRef]

19. Meneghetti, M.; Pecile, C. Charge-transfer organic crystals: Molecular vibrations and spectroscopic effects of electron-molecular vibration coupling of the strong electron acceptor TCNQF4. J. Chem. Phys. 1986, 84, 4149-4162. [CrossRef]

20. Girlando, A. Comment on Polymorphism in the 1:1 Charge-Transfer Complex DBTT-TCNQ and Its Effects on Optical and Electronic Properties. Adv. Electron. Mater. 2017, 3, 1600437. [CrossRef]

21. Frackowiak, A.; Olejniczak, I.; Świetlik, R.; Jeannin, O.; Fourmigué, M. Temperature-Induced Neutral-Ionic Phase Transition in the (EDT-TTF-I2)2TCNQF Mixed-Stack Charge-Transfer Salt. J. Phys. Chem. C 2016, 120, 23740-23747. [CrossRef]

22. Painelli, A.; Girlando, A. Electron-molecular vibration (e-mv) coupling in charge-transfer compounds and its consequences on the optical spectra: A theoretical framework. J. Chem. Phys. 1986, 84, 5655-5671. [CrossRef]

23. Masino, M.; Girlando, A.; Farina, L.; Brillante, A. A new type of neutral ionic interface in mixed stack organic charge transfer crystals: Temperature induced ionicity change in CIMePD DMeDCNQI. Phys. Chem. Chem. Phys. 2001, 3, 1904-1910. [CrossRef]

24. Maienschein-Cline, M.G.; Londergan, C.H. The CN stretching band of aliphatic thiocyanate is sensitive to solvent dynamics and specific solvation. J. Phys. Chem. A 2007, 111, 10020-10025. [CrossRef] [PubMed]

25. Zhu, W.; Yi, Y.; Zhen, Y.; Hu, W. Precisely tailoring the stoichiometric stacking of perylene-TCNQ co-crystals towards different nano and microstructures with varied optoelectronic performances. Small 2015, 11, 2150-2156. [CrossRef] [PubMed]

26. Hu, P.; Ma, L.; Tan, K.J.; Jiang, H.; Wei, F.; Yu, C.; Goetz, K.P.; Jurchescu, O.D.; McNeil, L.E.; Gurzadyan, G.G.; et al. Solvent-dependent stoichiometry in perylene-7,7,8,8-tetracyanoquinodimethane charge transfer compound single crystals. Cryst. Growth Des. 2014, 14, 6376-6382. [CrossRef]

27. Salzillo, T.; Della Valle, R.G.; Venuti, E.; Kociok-Köhn, G.; Masino, M.; Girlando, A.; Brillante, A. Solution equilibrium between two structures of Perylene- $\mathrm{F}_{2}$ TCNQ charge transfer co-crystals. J. Cryst. Growth 2019, 516, 45-50. [CrossRef]

28. Levina, E.O.; Chernyshov, I.Y.; Voronin, A.P.; Alekseiko, L.N.; Stash, A.I.; Vener, M.V. Solving the enigma of weak fluorine contacts in the solid state: A periodic DFT study of fluorinated organic crystals. RSC Adv. 2019, 9, 12520-12537. [CrossRef]

29. D'Avino, G.; Della Valle, R.G.; Heimel, G.; Oehzelt, M.; Kera, S.; Ueno, N.; Beljonne, D.; Salzmann, I. Electrostatic Interactions Shape Molecular Organization and Electronic Structure of Organic Semiconductor Blends. Chem. Mater. 2020. [CrossRef]

(C) 2020 by the authors. Licensee MDPI, Basel, Switzerland. This article is an open access article distributed under the terms and conditions of the Creative Commons Attribution (CC BY) license (http://creativecommons.org/licenses/by/4.0/). 